\title{
Visual Hallucinations from Zolpidem Use for the Treatment of Hospital Insomnia in a Septuagenarian
}

\author{
Raza Mian ${ }^{1}$ \\ 1. Internal Medicine, University of California San Francisco, Fresno, USA
}

Corresponding author: Raza Mian, raza.mian@outlook.com

\begin{abstract}
Insomnia and parasomnias are common patient complaints during hospital stay. New environment, the severity of underlying disease, level of care, medications, and infections are all known factors that contribute toward insomnia. Zolpidem is a common sleep aid used for this purpose. We report a case of Zolpidem-induced visual hallucination in a septuagenarian in the inpatient setting.
\end{abstract}

Categories: Family/General Practice, Internal Medicine, Neurology

Keywords: zolpidem, ambien, hallucination, insomnia

\section{Introduction}

Insomnia is a common complaint in the inpatient setting. Increasing age, disease severity, underlying psychiatric condition, pain, location, and medications contribute to insomnia. Therapies include nonpharmacological and pharmacological interventions. Sleep aids make the cornerstone of pharmacological interventions. The use of sleep aids in the inpatient setting is also common. These include sedating antihistamines, melatonin, antidepressants, and antipsychotics. Zolpidem is a commonly used sleep aid. We report a case of Zolpidem-induced visual hallucination in a patient in his seventies.

Received 12/18/2018 Review began 12/21/2018 Review ended 01/02/2019 Published 01/08/2019

\section{(c) Copyright 2019}

Mian. This is an open access article distributed under the terms of the Creative Commons Attribution License CC-BY 3.0., which permits unrestricted use, distribution, and reproduction in any medium, provided the original author and source are credited.

\section{Case Presentation}

A septuagenarian patient presented to the hospital with a complaint of worsening back pain. He had a pertinent previous medical history of recently diagnosed multiple myeloma. He reported sudden-onset, non-traumatic, spontaneous, mid-to-low, non-radiating, midline back pain that started two weeks ago. He denied association with bladder or bowel dysfunction, lower-extremity weakness, or paresthesia. His primary care provider had prescribed him non-steroidal anti-inflammatory agents without significant improvement. On presentation, he remained afebrile with normal vital signs. He had no saddle analgesia and a normal rectal tone. Post-void urinary bladder ultrasound revealed an empty bladder. He had a normal and symmetric sensation to light touch, vibration, and temperature. Patellar reflexes were normal and he had a normal Babinski's test. Hematological lab tests revealed microcytic anemia. Imaging studies revealed a compression fracture of the ninth and tenth thoracic vertebrae. Neurosurgery evaluated the patient recommending against surgical fixation. A spinal brace was provided to assist with comfort. Radiation oncology evaluated the patient and a decision was made to pursue radiation therapy.

The patient complained of insomnia during the hospital stay and was prescribed Zolpidem of $5 \mathrm{mg}$. At six o'clock, the next morning, the patient was noticed by nursing staff to be delirious reporting seeing graphic looking cats on the wall. Conservative measures and reorientation led to the subsequent resolution of the symptoms over a period of 10 minutes. The patient had complete recollection of the event. During the subsequent hospital stay, no further doses of Zolpidem were administered. No further episodes of visual hallucination occurred.

\section{Discussion}

Insomnia is a common patient complaint in the inpatient setting [1]. Various risk factors are known in this setting, which can contribute to insomnia, including disease severity, poor quality or amount of sleep, new environment, and disturbed wake-sleep cycle, lack of exercise, medications, and infections [2]. Hospitalacquired delirium shares some of the same risk factors [3]. Treatment is sought by employing nonpharmacological and pharmacological tools. These include improved daytime lighting including sunlight exposure, ambulation, reduced nighttime disturbance, and continued treatment of the underlying disease process [4]. However, many times, non-pharmacological aids are supplemented by pharmacological agents. Commonly employed agents include melatonin, sedative-hypnotics, antidepressants, and antipsychotic agents [5]. Zolpidem is a commonly used sedative-hypnotic.

The common side effects of Zolpidem include a headache, drowsiness, dizziness, lethargy, depression, and constipation. Severe side effects of Zolpidem include suicidal ideation, aggressive behavior, impaired mental 
alertness the following day, hallucinations, amnesia, anaphylaxis, angioedema, and withdrawal [6-9].

This case report discussed visual hallucination in a patient with risk factors associated with disturbed inpatient sleep and hospital delirium. Zolpidem was used as a sleep aid. Subsequently, hallucinations and delirium were noticed. Brodeur et al. reported a case of an octagenarian patient with a similar hallucination side effect; however, the symptoms lasted longer [10]. The impact on patient health during these episodes can be varied. There have been reports of somnambulism as well as homicide [11]. Advanced, female gender, concomitant use of selective serotonin reuptake inhibitors (SSRI), and Zolpidem dosage $>10 \mathrm{mg}$ have been reported to be associated with hallucinogenic side effects of Zolpidem; however, it can be seen in the younger population as well [10-13].

Although relatively safe, it is important to identify and recognize the potential side effects of this drug. Caution is advised in the at-risk population.

\section{Conclusions}

Insomnia is a common inpatient complaint. Symptomatic management with the use of sleep aids is common, and Zolpidem is a commonly used agent. Visual hallucinations can occur after Zolpidem administration. At-risk patients should avoid Zolpidem as a sleep agent.

\section{Additional Information \\ Disclosures}

Human subjects: Consent was obtained by all participants in this study. Conflicts of interest: In compliance with the ICMJE uniform disclosure form, all authors declare the following: Payment/services info: All authors have declared that no financial support was received from any organization for the submitted work. Financial relationships: All authors have declared that they have no financial relationships at present or within the previous three years with any organizations that might have an interest in the submitted work. Other relationships: All authors have declared that there are no other relationships or activities that could appear to have influenced the submitted work.

\section{References}

1. Badr AF, Kurdi S, Alshehri S, McManus C, Lee J: Pharmacists interventions to reduce sedative/hypnotic use for insomnia in hospitalized patients. Saudi Pharm J. 2018, 26:1204-1207. 10.1016/j.jsps.2018.07.010

2. Watson PL, Ceriana P, Fanfulla F: Delirium: is sleep important?. Best Pract Res Clin Anaesthesiol. 2012, 26:355-366. 10.1016/j.bpa.2012.08.005

3. Virginia BK, Joseph EG, Brian KU: Delirium in older persons: evaluation and management. Am Fam Physician. 2014, 90:150-158.

4. Tang NK, Lereya ST, Boulton H, Miller MA, Wolke D, Cappuccio FP: Nonpharmacological treatments of insomnia for long-term painful conditions: a systematic review and meta-analysis of patient-reported outcomes in randomized controlled trials. Sleep. 2015, 38:1751-64. 10.5665/sleep.5158

5. Sateia MJ, Buysse DJ, Krystal AD, Neubauer DN, Heald JL: Guideline for the pharmacologic treatment of chronic insomnia in adults: an American Academy of Sleep Medicine clinical practice guideline. J Clin Sleep Med. 2017, 13:307-349. 10.5664/jcsm.6470

6. Wong CK, Marshall NS, Grunstein RR, et al.: Spontaneous adverse event reports associated with zolpidem in the United States 2003-2012. J Clin Sleep Med. 2017, 13:223-234.

7. Singh G, Loona N: Zolpidem-induced hallucinations: a brief case report from the Indian subcontinent . Indian J Psychol. 213, 35:212-213.

8. Tsai MJ, Huang YB, Wu PC: A novel clinical pattern of visual hallucination after zolpidem use . J Toxicol Clin Toxicol. 2003, 41:869-872.

9. Ganzoni E, Santoni J, Chevillard V, Sébille M, Mathy B: Zolpidem in insomnia: a 3-year post-marketing surveillance study in Switzerland. J Int Med Res. 1995, 23:61-73.

10. Brodeur MR, Stirling AL: Delirium associated with zolpidem. Ann Pharmacother. 2001, 35:1562-4. 10.1345/aph.10385

11. Paradis CM, Siegel LA, Kleinman SB: Two cases of zolpidem-associated homicide. Prim Care Companion CNS Disord. 2012, 14:10.4088/PCC.12br01363

12. Inagaki T, Miyaoka T, Tsuji S, Inami Y, Nishida A, Horiguchi J: Adverse reactions to zolpidem: case reports and a review of the literature. Prim Care Companion J Clin Psychiatry. 2010, 12:e1-e8. 10.4088/PCC.09r00849bro

13. Ram D, Eiman N, Gowdappa B: Multimodal hallucination (audio-visual, kinaesthetic and scenic) associated with the use of zolpidem. Clin Psychopharmacol Neurosci. 2015, 13:215-217. 10.9758/cpn.2015.13.2.215 